DePauw University

Scholarly and Creative Work from DePauw University

$9-10-2016$

\title{
Facilitating Emotional Regulation: The Interactive Effect of Resource Availability and Reward Processing
}

Michael E. Roberts

DePauw University, michaelroberts@depauw.edu

Follow this and additional works at: https://scholarship.depauw.edu/psyc_facpubs

Part of the Social Psychology Commons

\section{Recommended Citation}

(C) 2016. This manuscript version is made available under the CC-BY-NC-ND 4.0 license http://creativecommons.org/licenses/by-nc-nd/4.0/ Published version in: Roberts, M. E., Clarkson, J. J., Cummings, E. L., \& Ragsdale, C. M. (2016). Facilitating emotional regulation: The interactive effect of resource availability and reward processing. Journal Of Experimental Social Psychology, doi:10.1016/ j.jesp.2016.09.002

This Article is brought to you for free and open access by the Psychology and Neuroscience at Scholarly and Creative Work from DePauw University. It has been accepted for inclusion in Psychology and Neuroscience Faculty Publications by an authorized administrator of Scholarly and Creative Work from DePauw University. 
RUNNING HEAD: Facilitating Emotional Regulation

\title{
Facilitating Emotional Regulation:
}

\section{The Interactive Effect of Resource Availability and Reward Processing}

\author{
Michael E. Roberts \\ DePauw University \\ Joshua J. Clarkson \\ University of Cincinnati \\ Elizabeth L.T. Cummings \\ DePauw University \\ Chelsea M. Ragsdale \\ DePauw University
}

Word Count: 3912

\section{Correspondence.}

Michael E. Roberts, $\mathrm{PhD}$

Associate Professor of Psychology

DePauw University

Greencastle, IN, 46135

michaelroberts@depauw.edu 


\section{Highlights}

- Resource availability and reward interact to impact the efficacy of emotion regulation.

- High resource availability facilitates the effect of reward on emotion regulation.

- Low resource availability inhibits the effect of reward on emotion regulation.

- This pattern holds only for high intensity rewards. 
Despite a wealth of knowledge on the importance of resource availability and reward processing for emotional regulation, surprisingly little is known about the extent to which these two mechanisms interact. Indeed, while research largely supports a positive association between reward processing and recovering from a negative emotional experience, the research does not make a clear prediction regarding the effect of resource availability on this relationship. In two experiments, we explored the extent to which resource availability impacts the efficacy of reward processing to reduce the aversive emotional experience of anxiety. We manipulated participants' mental resource availability, induced anxiety, and varied exposure to either a rewarding or nonrewarding stimulus. The findings consistently demonstrate an interaction between resource availability and reward processing; specifically, the combination of high resource availability and reward processing facilitated the greatest levels of anxiety reduction. Moreover, this interaction was shown to amplify with the intensity of participants' exposure to the reward stimulus. We discuss the practical contributions of these findings and their generative nature for further clarifying the processes underlying emotional regulation.

Keywords: emotional regulation, cognitive control, mental resources, reward processing, selfcontrol, resource depletion, mental fatigue, anxiety 
Broadly defined, emotional regulation can refer to a variety of strategies that involve heightening, maintaining, coping with, changing, or recovering from an emotional experience (Ochsner \& Gross, 2005; Gyurak, Gross, \& Etkin, 2011). It is not surprising that emotional regulation utilizes mental resources (Muraven, Tice, \& Baumeister, 1998; Pe et al., 2015; Schmeichel, Volokhov, \& Demaree, 2008). For instance, researchers have repeatedly linked mental resource availability to the successful suppression of negative emotions (Schmeichel et al., 2008; Unsworth, Heitz, \& Engle, 2005; Pe et al., 2015). Furthermore, the lack of available mental resources can weaken a critical emotional regulation pathway and thereby impact individuals' recovery from adverse emotional states (Muraven et al., 1998; Wagner \& Heatherton, 2013). ${ }^{1}$

Mental resources may also have more nuanced effects on emotional regulation. Consistent with a balance model of self-regulation, the lack of available mental resources has been shown to weaken the regulation of striatal brain regions associated with reward processing and may allow cravings to further interfere with self-regulation (Kober et al., 2010; Wagner, Altman, Boswell, Kelley, \& Heatherton, 2013; Giuliani, Mann, Tomiyama, \& Berkman, 2014). For example, chronic dieters who viewed appetizing food images when low (versus high) in resource availability showed greater activation in a reward processing region (orbitofrontal cortex) and less functional connectivity to a mental resource region (inferior frontal gyrus) (Wagner et al., 2013).

These effects of resource availability on reward processing are potentially significant for emotional regulation. Whereas much of the relevant research on depleted resource availability suggests that undesirable rewards and urges can lead to spiraling negative emotional regulation consequences, a small number of emotional regulation studies indicate that positive reward 
processing can be beneficial to emotional regulation. For example, listening to pleasant music is associated with activation of reward-processing regions (Blood, Zatorre, Bermudez, \& Evans, 1999) and was found to significantly reduce negative emotions and physiological arousal (Labbé, Schmidt, Babin, \& Pharr, 2007). Similarly, several cognitive neuroscience studies show that activation of a pathway between prefrontal cortex cognitive control regions and ventral striatum correlates with successful reappraisal, one of the most effective and well-studied emotional regulation strategies (Wager, Davidson, Hughes, Lindquist, \& Ochsner, 2008; McRae et al., 2010; Buhle et al., 2013). The ventral striatum is often associated with reward processing, and the pathway is thought to be involved in the processing of positive appraisals. However, these studies do not manipulate individuals' available mental resources, so it is unclear if the reward processing would still facilitate emotional regulation for individuals who lack available resources.

The present work provides a direct behavioral test of a potential interaction between resource availability and reward processing on emotional regulation. The aforementioned studies agree that a lack of available mental resources impairs emotional regulation; however, the role of reward is unclear, as the studies that find a positive association with emotional regulation do not consider possible interactions with mental resources. Consequently, we test three interaction hypotheses that can be drawn from extant literature.

First, when mental resources are unavailable, a reward stimulus will harm emotional regulation. In support of this hypothesis, regulation of reward processing may require the same resources needed for emotional regulation (e.g., Kober et al., 2010). Second, when mental resources are unavailable, a reward stimulus will facilitate emotional regulation. In support of this hypothesis, a lack of available resources can increase attention towards rewards 
(Schmeichel, Harmon-Jones, \& Harmon-Jones, 2010) which may, in turn, reduce negative emotions (Labbé et al., 2007). Third, when mental resources are available, a reward stimulus will facilitate emotional regulation. In support of this hypothesis, the aforementioned neuroimaging emotional regulation studies found a relationship between reward processing regions and emotional regulation, and none of those studies reduced participants' resource availability through experimental manipulation (Wager et al., 2008; McRae et al., 2010; Buhle et al., 2013).

Two experiments directly test these hypotheses by manipulating resource availability and the presence or absence of a rewarding stimulus to assess their interactive impact on emotional regulation. Three aspects of our procedure are worth noting. First, we focus on anxiety as a representative negative emotional experience, as numerous studies indicate that anxiety activates a negative appraisal pathway between prefrontal cortex regions and the amygdala (e.g., Ochsner et al., 2004; Banks, Eddy, Angstadt, Nathan, \& Phan, 2007; Wager et al., 2008; Heatherton, 2011). Importantly, then, we controlled for participants' dispositional tendencies to experience anxiety using the trait anxiety subscale of the State-Trait Anxiety Inventory-Y (Spielberger, Gorsuch, \& Lushene, 1970) as well as participants' gender, as prior research shows differences in experienced anxiety across males and females (Feingold, 1994). Second, we did not provide instructions for a specific emotional regulation strategy; instead, we expected participants to pursue spontaneous emotional regulation (Egloff, Schmukle, Burns, \& Schwerdtfeger, 2006; Berkman \& Lieberman, 2009). Third, the sample size in Experiment 2 was determined by those used in prior experiments on mental resource availability (e.g., Clarkson, Hirt, Jia, \& Alexander, 2010; Muraven, Shmueli, \& Burkley, 2006), whereas lab demand led to a higher than expected 
sample size for Experiment 1. Informed consent was obtained from all participants, and all measures, manipulations, and exclusions are reported.

\section{Experiment 1}

We conducted an initial experiment to assess the interactive effect of resource availability and reward processing on anxiety reduction. Our manipulation of reward stimulus allowed us to test the role of reward valence by including a control (i.e., no reward) condition.

\section{Method}

Participants and Design.

Three hundred and twenty-six undergraduates $\left(54 \%\right.$ Female; $\left.M_{\mathrm{age}}=20.28\right)$, participating for course credit, were randomly assigned to conditions in a 2 (Resource Availability: High or Low) $\times 3$ (Music Exposure: Classical, Heavy Metal, or Silent) between-participant design. Procedure.

Participants were welcomed to the lab where they completed our demographic and STAI$\mathrm{T}(\alpha=.90)$ background measures. We then exposed participants to our manipulation of resource availability, which we described as a test of cognitive acuity. The task presented participants with a series of six multiple-solution anagrams before being informed that cognitive acuity is either best assessed on this task when respondents pay close attention to the task (low resource availability) or when respondents complete the task as they would normally (high resource availability). Participants were further told they would have twenty-seconds to generate as many words as possible for each anagram. This manipulation was based on similar instructional sets used to vary resource availability (Baumeister, Bratslavsky, Muraven, \& Tice, 1998), as attentional demands reduce resource availability (Baumeister et al., 1998; Clarkson et al., 2010; Muraven et al., 2006). 
Following the manipulation of resource availability, participants indicated their current level of mental fatigue on the four-item mental fatigue subscale of the Multidimensional Fatigue Inventory (Smets, Garssen, Bonke, \& De Haes, 1995). This subscale assesses one's ability to engage in mental activity (e.g., "It takes a lot of effort to concentrate on things right now."). Responses were obtained on 5-point scales labeled 1 - Not at all true to 5 - Very true, and averaged $(\alpha=.77)$ to form a composite index of mental fatigue, with higher scores indicating greater mental fatigue. This measure served as a manipulation check of resource availability (see Clarkson, Hirt, Chapman, \& Jia, 2011).

Participants then completed the anxiety induction task, which was presented as an empathy exercise that described a series of three high anxiety scenarios (e.g., imagine receiving a pop test worth $30 \%$ of your course grade). Participants were exposed to each scenario for two minutes, during which they were instructed to experience the scenario as though it were real and to focus on the emotions they would feel. These scenarios were pretested to elicit anxiety and thus served as our situational induction of anxiety given that ruminating on anxiety-provoking stimuli has been shown to heighten the actual experience of anxiety (Tesser, Leone, \& Clary, 1978).

After the final scenario, we assessed participants' initial state anxiety using the state anxiety subscale of the STAI-Y (Spielberger et al., 1970). The subscale focuses on the extent to which individuals are currently experiencing anxiety-related symptoms. Responses were obtained on 4-point scales labeled 1 -almost never, 2 - sometimes, 3 -often, and 4-almost always and averaged $(\alpha=.96)$ such that higher scores indicated greater initial state anxiety.

Finally, participants were randomly assigned to listen to one of three music samples as our manipulation of reward. Specifically, participants listened to ten minute clips of classical 
music, heavy metal music, or silence. Classical music has been shown to activate striatal reward processing regions (e.g. Salimpoor, Benovoy, Larcher, Dagher, \& Zatorre, 2011; Salimpoor et al., 2013), and prior research demonstrates that classical music induces greater reward processing compared to both heavy metal music and silence (Blood, Zatorre, Bermudez, \& Evans, 1999; Brown, Martinez, \& Parsons, 2004; Koelsch, Fritz, Yves v. Cramon, \& Muller, 2006; Labbé et al., 2007).

Afterward, participants again completed the state anxiety subscale of the STAI-Y $(\alpha=$ .93) before being debriefed and thanked for their participation.

\section{Results}

All measures were submitted to a 2 (Resource Availability: High or Low) $\times 3$ (Music Exposure: Classical, Heavy Metal, or Silent) ANOVA, with participants' trait anxiety and gender as covariates. $^{2,3}$

Mental Fatigue.

Analysis of the mental fatigue index revealed a significant main effect of resource availability $\left(F(1,318)=10.53, p=.001, \eta_{p}^{2}=.03\right)$ in the expected pattern; those in the low availability condition $(M=2.21, S D=.76)$ reported greater mental fatigue than did those in the high availability condition $(M=1.96, S D=.79)$. Neither the main effect of music nor the interaction was significant (all $F \mathrm{~s}<1)$.

Anxiety Reduction.

An anxiety reduction score was created by subtracting participants' post-music state anxiety from their pre-music state anxiety. Analysis of this score revealed a significant main effect of music exposure $\left(F(2,318)=8.60, p<.001, \eta_{p}^{2}=.05\right)$ that was qualified by a resource availability $\times$ music exposure interaction $\left(F(2,318)=7.83, p<.001, \eta_{p}^{2}=.05\right)($ see Figure 1$)$. 
For those high in available resources, there was a significant main effect of music exposure $(\mathrm{F}(2,318)=18.84, p<.001)$; replicating prior research that did not manipulate participants' resource availability (Labbé et al., 2007), exposure to classical music generated greater anxiety reduction than did exposure to either heavy metal music $(t(174)=6.22, p<.001)$ or silence $(t(174)=4.50, p<.001)$, which marginally differed from each other $(t(174)=1.69, p=.092)$. For those low in available resources, there was no effect of music exposure on anxiety reduction $(F<1){ }^{4}$

\section{Discussion}

Experiment 1 provided evidence that activation of reward processing (here, classical music) generated greater anxiety reduction for those with available regulatory resources. Indeed, this heightened anxiety reduction in the classical music condition was greater than both the heavy metal music and silent conditions. Those in the low resource availability condition, however, showed no difference in anxiety reduction as a function of reward activation. This finding suggests that resource availability increases the impact of reward processing on emotional regulation.

It is interesting to note that, for those with available mental resources, the activation of reward (i.e., classical music) not only facilitated anxiety reduction following exposure to classical music but appeared to decelerate anxiety reduction following exposure to heavy metal music. This deceleration effect suggests that the availability of mental resources heightens individuals' sensitivity to any new appraisals (positive in the case of the classical music condition and negative in the case of the heavy metal music condition). We revisit this point in the General Discussion.

\section{Experiment 2}


The initial experiment indicates that resource availability and reward processing can interact to facilitate emotional regulation. In a second experiment, we sought to test the robustness of this effect while examining the importance of the intensity of exposure to the reward stimulus. Specifically, in Experiment 1, we exposed participants to ten minutes of music. However, in neuroimaging studies, even relatively brief exposure to musical pieces has been linked to activity in reward processing regions (e.g., music clips had durations of 30 seconds in Salimpoor et al, 2013; 45-60 seconds in Koelsch et al., 2006; 60 seconds in Brown et al., 1999; approximately 73 seconds in Blood et al., 1999). But the likelihood and intensity of reward activation appears to build as a participant listens to longer clips (e.g., 3 minutes in Salimpoor, et al., 2011). Thus, we explored whether any exposure to reward is sufficient for the interaction found in Experiment 1 or whether the duration of the exposure need be appropriately intense for reward processing to interact with resource availability to determine emotional regulation.

Thus, in Experiment 2, we used the same reward stimuli as Experiment 1 but varied the duration of exposure to a stimulus (i.e., classical or heavy metal music) as our manipulation of reward intensity - that is, the degree to which reward processing has been activated by the stimulus. We hypothesized that the interaction between resource availability and reward exposure would only occur when the reward is sufficiently strong (i.e. high intensity), as more intense exposure should result in greater reward activity (see Salimpoor et al., 2011). Additionally, Experiment 2 also included a baseline anxiety measure in order to ensure participants' state anxiety was equal prior to our anxiety manipulation, and we used alternative manipulations of resource availability and anxiety induction to test the robustness of the interaction.

\section{Method}


Participants and Design.

One hundred and thirty-seven undergraduates $\left(58 \%\right.$ Female; $\left.M_{\text {age }}=20.00\right)$, participating for course credit, were randomly assigned to conditions in a 2 (Resource Availability: High or Low $) \times 2$ (Music Exposure: Classical or Heavy Metal) $\times 2$ (Reward Intensity: High or Low) between-participant design.

Procedure.

As in Experiment 1, participants were welcomed to the lab where they completed our demographic and STAI-T $(\alpha=.87)$ background measures. Here we also included a baseline assessment of their state anxiety using the state anxiety subscale of the STAI-Y $(\alpha=.92)$ to ensure that the anxiety induction task was sufficiently potent. We next exposed participants to our manipulation of resource availability. This manipulation consisted of 200 trials of either an "incongruent" four-color Stroop task in which the background color mismatched the presented word (low resource availability) on all trials or a "congruent" four-color Stroop task in which the background color matched the presented word (high resource availability) on all trials. This manipulation is based on prior research demonstrating that the attention required to respond to incongruent (versus congruent) trials decreases the availability of mental resources (see Inzlicht, McKay, \& Aronson, 2006). Upon completing this manipulation, participants indicated their level of perceived mental fatigue as in Experiment $1(\alpha=.81)$.

We then induced situational anxiety in all participants by informing them that they would be asked to prepare and then deliver a speech to be evaluated by experimenters at a later point in time. Specifically, they were informed they would be discussing what they liked and disliked about their body. They were then given one minute to prepare their response and three minutes to write an essay on the topic. This induction was adapted from prior research used to successfully 
induce anxiety (Phillips \& Giancola, 2008). Upon completing the essay, participants indicated their current level of anxiety using the state anxiety subscale of the STAI-Y $(\alpha=.93)$.

As in Experiment 1, participants were randomly assigned to listen to music clips of either classical or heavy metal music as our manipulation of reward. Here, however, we also varied the intensity of reward activation by altering the duration of music exposure. In the high intensity condition, participants were exposed to ten minutes of music. In the low intensity condition, participants were exposed to thirty seconds of music.

Afterwards, participants again completed the state anxiety subscale of the STAI-Y $(\alpha=$ .94) before being debriefed and thanked for their participation.

\section{Results}

All measures were submitted to a 2 (Resource Availability: High or Low) $\times 2$ (Music Exposure: Classical or Heavy Metal) $\times 2$ (Reward Intensity: High or Low) ANOVA, with participants' trait anxiety and gender as covariates.

\section{Mental Fatigue.}

Analysis of the mental fatigue index revealed a trending effect of resource availability manipulation $\left(F(1,127)=1.78, p=.18, \eta_{p}^{2}=.014\right)$. Consistent with Experiment 1 , those in the low availability condition $(M=2.37, S D=1.06)$ reported greater mental fatigue than did those in the high availability condition $(M=2.05, S D=.83)$. There was also a trending music exposure $\times$ reward intensity interaction, $F(1,127)=2.40, p=.12, \eta_{p}^{2}=.02$, but no other effects were significant (all $p \mathrm{~s}>.35)$.

\section{Anxiety Reduction.}

As in Experiment 1, an anxiety reduction score was created by subtracting participants' post-music state anxiety from their pre-music state anxiety. Analysis of this score revealed a 
significant main effect of music exposure $\left(F(1,127)=17.68, p<.001, \eta_{p}^{2}=.12\right)$ that was qualified by a resource availability $\times$ music exposure $\times$ reward intensity interaction $(F(1,127)=$ $4.28, p=.041, \eta_{p}^{2}=.03$ ) (see Figure 2). No other effect was significant (all $p \mathrm{~s}>.12$ ). We elected to break this three-way interaction down by reward intensity.

For those exposed to 10 minutes of music (i.e., the high intensity condition), the main effect of music exposure was significant $\left(F(1,65)=12.62, p=.001, \eta^{2}{ }_{p}=.16\right)$; however, this main effect was qualified by a resource availability $\times$ music exposure interaction $(F(1,65)=8.27$, $\left.p=.005, \eta_{p}^{2}=.11\right)$. For participants high in resource availability, exposure to the classical (versus heavy metal) music led to significantly greater anxiety reduction $(F(1,65)=19.09, p<$ $.001, \eta_{p}^{2}=.23$ ). For individuals low in resource availability, music exposure had no effect on anxiety reduction $(F<1)$. These results are consistent with the findings of Experiment 1 .

For those exposed to 30 seconds of music (i.e., the low intensity condition), only the main effect of music exposure was significant $\left(F(1,60)=6.78, p=.012, \eta_{p}^{2}=.10\right)$. Neither the main effect of resource availability nor the resource availability $\times$ music exposure interaction was significant $(p s>.23)$.

\section{Discussion}

The findings of Experiment 2 illustrate the importance of reward intensity on the interaction between resource availability and reward processing. As in Experiment 1, exposure to a reward stimulus (classical music) led to significant anxiety reduction when participants had available mental resources. However, this effect emerged only in the high intensity condition; those in the low intensity condition showed only a significant main effect of reward processing. These findings suggest that reward intensity is a critical determinant for when resource availability will interact with reward processing to determine anxiety regulation. Importantly, 
the duration of the reward condition is not critical by itself, as the 10-minute heavy metal condition did not lead to comparable benefits in either experiments nor did the 10-minute silent condition in Experiment 1.

Two additional results of this experiment are worth noting. First, these results demonstrate that some anxiety reduction occurs for the low resource availability conditions even without exposure to a rewarding stimulus, a result that appears more consistent for the longer duration. This finding lends tentative support to a homeostatic mechanism with ebbing over time (i.e., a natural return to low anxiety as time increases). Second, as in the previous experiment, the results also hint that resource availability increases sensitivity to the stimulus, regardless of whether the stimulus is positive or negative.

\section{General Discussion}

We began with three hypotheses for an interactive relationship between resource availability and reward processing in driving anxiety regulation. Across two experiments, the findings provide consistent and convergent support for the third hypothesis: Individuals with available resources who engage in reward processing demonstrated a significantly greater reduction in anxiety. This interaction was robust across different manipulations of resource availability and different inductions of anxiety. Additionally, neither trait anxiety nor participant sex moderated the interaction effect in the experiments (see Footnote 2) nor did the pattern of anxiety change when directly analyzing time 2 anxiety scores (controlling for initial anxiety) (see Footnote 4). Moreover, the interaction only occurred when the intensity of the reward was sufficient; when insufficient, only a main effect of reward processing was observed.

As noted, previous studies have found a positive relationship between reward processing and emotional regulation. However, none of those studies addressed the role of resource 
availability (e.g. Wager et al., 2008, McRae et al., 2010; Buhle et al., 2013). Our experiments replicate this positive relationship but demonstrate it is bounded to conditions of high resource availability. Indeed, under low resource availability, reward processing was shown to have no impact on anxiety reduction. This null effect is particularly striking given participants were exposed to the reward stimulus for ten minutes. ${ }^{5}$

Importantly, we found no support for the alternative hypotheses that individuals low in resource availability would be negatively affected by reward processing (Hypothesis 1) or, conversely, that participants low in resource availability would focus attention on rewards and experience greater anxiety reduction (Hypothesis 2). Yet the lack of support for these hypotheses does not mean these effects could not occur in some circumstances. For example, in regards to the first hypothesis, fulfilling a craving (e.g. for food, cigarettes, etc.) may stimulate reward processing but promote additional negative emotions and make anxiety increasingly difficult to regulate with limited available mental resources. In our experiments, the reward stimuli were benignly positive.

\section{Practical Implications.}

The lack of available mental resources can enhance cravings (Wagner et al., 2012) and perhaps even drive individuals towards popular coping mechanisms such as comfort eating or smoking (Kober et al., 2010). Yet the present findings suggest that rewards only become useful for emotional regulation when individuals possess available mental resources. Thus, if individuals do not already implicitly use this mechanism for emotional regulation, then explicit instruction may have practical benefits. It may also be fruitful to test clinical applications of this interaction between resource availability and reward processing, as repeatedly utilizing the shortterm benefits of this emotional regulation mechanism (see Denny, Inhoff, Zerubavel, Davachi, \& 
Ochsner, 2015) may supplement existing treatments for chronic anxiety (Abramowitz, Deacon \& Whitseide, 2011; Ferrari, Lapp, \& Perretti, 2011; Wolpe, 1997).

\section{Future Directions.}

Additional work will be needed to elucidate the mechanism behind the interaction. As we noted in the discussion sections of both experiments, the availability of mental resources appeared to make participants more sensitive to positive and negative reward processing. However, this sensitivity could result from independent effects. For instance, positive reward processing interacts with available mental resources to facilitate emotional regulation, whereas negative reward processing may draw upon the lack of available mental resources and thereby hinder emotional regulation. Similarly, the magnitude of reward activation necessary to facilitate emotional regulation may depend on the magnitude of the initial negative appraisal. Indeed, with stronger anxiety manipulations, resource availability may have a larger role in shifting and enhancing appraisals, but reward activation may also also need to be proportionately stronger in order to be beneficial.

\section{Conclusion.}

In two experiments, resource availability and reward processing interacted to facilitate anxiety reduction after an anxiety-inducing event; specifically, activation of reward processing generated greater anxiety reduction for those with available regulatory resources. Reward processing was not beneficial when participants were low in resource availability. Moreover, this effect depends on the intensity of the rewarding stimulus and is independent of gradual anxiety reduction that appears to occur in all conditions. Consequently, these results offer direct insight into the interactive role of both resource availability and reward processing for emotional 
regulation and, as such, should stimulate novel predictions concerning when and why individuals are able to successfully engage in emotional regulation. 


\section{Compliance with Ethical Standards}

Ethical approval: All procedures performed in studies involving human participants were in accordance with the ethical standards of the institutional and/or national research committees and with the 1964 Helsinki declaration and its later amendments or comparable ethical standards.

Informed consent: Informed consent was obtained from all individual participants included in the studies, including the three experiments and supplementary experiment. 


\section{References}

Abramowitz J. S., Deacon, B. J., \& Whiteside, S. P. H. (2011). Exposure therapy for anxiety: Principles and practice. New York: Guildford Press.

Banks, S.J. Eddy, K.T., Angstadt, M., Nathan, P.J., \& Phan, K.L. (2007) Amygdala frontal connectivity during emotion regulation. Social Cognitive and Affective Neuroscience, 2, $303-312$.

Baumeister, R. F., Bratslavsky, E., Muraven, M., \& Tice, D. M. (1998). Ego depletion: Is the active self a limited resource? Journal of Personality and Social Psychology, 74(5), 1252-1265.

Berkman, E. T., \& Lieberman, M.D. (2009). Using neuroscience to broaden emotion regulation: Theoretical and methodological considerations. Social and Personality Psychology Compass, 4, 475-493.

Blood, A.J., Zatorre, R.J., Bermudez, P., Evans, A.C. (1999). Emotional responses to pleasant and unpleasant music correlate with activity in paralimbic brain regions. Nature Neuroscience, 2, 382-387.

Brown, S., Martinez, M.J., Parsons, L.M. (2004). Passive music listening spontaneously engages limbic and paralimbic systems. NeuroReport, 15, 2033-2037.

Buhle, J.T., Silvers, J.A., Wager, T.D., Lopez, R., Onyemekwu, C., Kober, H., Weber, J., \& Ochsner, K.N. (2013). Cognitive reappraisal of emotion: A meta-analysis of human neuroimaging studies. Cerebral Cortex, 24, 2981-2990.

Clarkson, J.J., Hirt, E.R., Chapman, D.A., \& Jia, L. (2011). The impact of illusory fatigue on executive control: Do perceptions of depletion impair working memory capacity? Social Psychological and Personality Science, 2, 229-238. 
Clarkson, J.J., Hirt, E.R., Jia, L., \& Alexander, M.B. (2010). When perception is more than reality: The effect of perceived versus actual resource depletion on self-regulatory behavior. Journal of Personality and Social Psychology, 98, 29-46.

Denny, B.T., Inhoff, M.C., Zerubavel, N., Davachi, L., \& Ochsner, K.N. (2015). Getting over it: Long-lasting effects of emotion regulation on amygdala response. Psychological Science, 26, 1377-1388.

Egloff, B., Schmukle, S.C., Burns, L.R., \& Schwerdtfeger, A. (2006). Spontaneous emotion regulation during evaluated speaking tasks: Associations with negative affect, anxiety expression, memory, and physiological responding. Emotion, 6, 356-366.

Feingold, A. (1994). Gender differences in personality: A meta-analysis. Psychological Bulletin, $116,429-456$.

Ferrari, F., Lapp, L. K., \& Peretti, C-S. (2011). Current research on cognitive aspects of anxiety disorders. Current Opinion in Psychiatry, 24, 49-54.

Giuliani, N.R., Mann, T., Tomiyama, A.J., \& Berkman, E.T. (2014). Neural systems underlying the reappraisal of personally craved foods. Journal of Cognitive Neuroscience, 26, 1390-1402.

Gyurak, A., Gross, J.J. and Etkin, A. (2011). Explicit and implicit emotion regulation: A dualprocess framework, Cognition \& Emotion, 25, 400-412.

Heatherton, T.F., Wagner, D.D. (2011). Cognitive neuroscience of self-regulation failure. Trends in Cognitive Science, 15, 132-139.

Hirt, E.R., Clarkson, J.J., \& Jia, L. (Eds.). (2016). Self-Regulation and Ego Control. San Diego, CA: Elselvier. 
Inzlicht, M., McKay, L., \& Aronson, J. (2006). Stigma as ego depletion: How being the target of prejudice affects self-control. Psychological Science, 17, 262-269.

Inzlicht, M., \& Schmeichel, B.J. (2012). What is ego depletion? Toward a mechanistic revision of the resource model of self-control. Perspectives on Psychological Science, 7(5), 450-463.

Kober, H., Mende-Siedlecki, P., Kross, E.F., Weber, J., Mischel, Q., Hart, C.L., \& Ochsner, K.N. (2010). Prefrontal-striatal pathway underlies cognitive regulation of craving. Proceedings of the National Academy of Sciences, 3, 14811-14816.

Koelsch, S., Fritz, T., Yves v. Cramon, D., Muller, K., Friederici, A.D. (2006). Investigating emotion with music: An fMRI study. Human Brain Mapping, 27, 239-250.

Kotabe, H.P., \& Hofmann, W. (2015). On integrating the components of self-control. Perspectives on Psychological Science, 10(5), 618-638.

Labbé, E., Schmidt, N., Babin, J., Pharr, M. (2007). Coping with stress: The effectiveness of different types of music. Applied Psychophysiology \& Biofeedback, 32, 163-168.

McRae, K., Hughes, B., Chopra, S., Gabrieli, J.D.E., Gross, J.J., \& Ochsner, K.N. (2010). The neural bases of distraction and reappraisal. Journal of Cognitive Neuroscience, 22, 248-262.

Molden, D.C., Hui, C.M., Scholer, A.A., Meier, B.P., Noreen, E.E., D’Agostino, P.R., \& Martin, V. (2012). Motivational versus metabolic effects of carbohydrates on self-control. Psychological Science, 23, 1137-1144.

Muraven, M., Shmueli, D. \& Burkley, E. (2006). Conserving self-control strength. Journal of Personality and Social Psychology, 91, 524-537. 
Muraven, M., Tice, D.M., \& Baumeister, R.F. (1998). Self-control as limited resource: Regulatory depletion patterns. Journal of Personality and Social Psychology, 74, 774789.

Ochsner, K.N. \& Gross, J.J. (2005). The cognitive control of emotion. Trends in Cognitive Science, 9, 242-249.

Ochsner, K.N., Ray, R. D., Cooper, J.C., Robertson, E.R., Chopra, S., Gabrieli, J.D., \& Gross, J.J. (2004). For better or for worse: neural systems supporting the cognitive down- and up-regulation of negative emotion. Neuroimage, 23, 483-499.

Pe, M.L., Koval, P., Houben, M., Erbas, Y., Champagne, D., \& Kuppens, P. (2015). Updating in working memory predicts greater emotion reactivity to and facilitated recovery from negative emotion-eliciting stimuli. Frontiers in Psychology, 6(372). doi: 10.3389/fpsyg.2015.00372

Phillips, J.P., \& Giancola, P.R. (2008). Experimentally induced anxiety attenuates alcoholrelated aggression in men. Experimental and Clinical Psychopharmacology, 16, 43-56.

Salimpoor, V.N., Benovoy, M., Larcher, K., Dagher, A., Zatorre, R.J. (2011). Anatomically distinct dopamine release during anticipation and experience of peak emotion to music. Nature Neuroscience, 14, 257-264.

Salimpoor, V.N., van den Bosch, I., Kovacevic, N., McIntosh, A.R., Dagher, A., \& Zatorre, R.J. (2013). Interactions between the nucleus accumbens and auditory cortices predict music reward value. Science, 340, 215-219.

Schmeichel, B.J., Harmon-Jones, C., \& Harmon-Jones, E. (2010). Exercising self-control increases approach motivation. Journal of Personality and Social Psychology, 99, $162-$ 173. 
Schmeichel, B.J., Volokhov, R.N., \& Demaree, H.A. (2008). Working memory capacity and the self-regulation of emotional expression and experience. Journal of Personality and Social Psychology, 95, 1526-1540.

Smets, E.M.A., Garssen, B., Bonke, B., De Haes, J.C.J. (1995). The multidimensional fatigue inventory (MFI) psychometric qualities of an instrument to assess fatigue. Journal of Psychosomatic Research, 39, 315-325.

Spielberger, C. D., Gorsuch, R.L., \& Lushene. R.E. (1970). Manual for the State-Trait Anxiety Inventory. Palo Alto, CA: Consulting Psychologists.

Tesser, A., Leone, C., \& Clary, E. G. (1978). Affect control: process constraints versus catharsis. Cognitive Therapy \& Research, 2, 265-274.

Unsworth, N., Heitz, R. P., \& Engle, R. W. (2005). Working memory capacity in hot and cold cognition. In R. W. Engle, G. Sedek, U. Hecker, \& D. N. McIntosh (Eds.), Cognitive limitations in aging and psychopathology (pp. 19-43). New York: Cambridge University Press.

Wager, T.D., Davidson, M.L., Hughes, B.L., Lindquist, M.A., \& Ochsner, K.N. (2008). Prefrontal-subcortical pathways mediating successful emotion regulation. Neuron, 59, $1037-1050$.

Wagner, D.D., Altman, M., Boswell, R.G., Kelley, W.M., \& Heatherton, T.F. (2013). Selfregulatory depletion enhances neural responses to rewards and impairs top-down control. Psychological Science, 24, 2262-2271.

Wagner, D.D., \& Heatherton, T.F. (2013). Self-regulatory depletion increases emotional reactivity in the amygdala. Social Cognitive and Affective Neuroscience, 8, 410-417. 
Wolpe, J. (1997). From psychoanalytic to behavioral methods in anxiety disorders: A continuing evolution. In J. K. Zeig (Ed.), The evolution of psychotherapy: The third conference. New York: Brunner/Mazel. 
FACILITATING EMOTIONAL REGULATION 26

\section{Footnotes}

1. The literature review in this domain focuses on effects in which emotional regulation is improved when individuals have access to mental resources. As such, we describe this access as stemming from the availability of mental resources to be consistent with recent perspectives that these resources are differentially allocated and thus differentially available based on various factors such as motivation (Inzlicht and Schmeichel, 2012; Molden et al., 2012, 2016), perception (Clarkson, Hirt, Chapman, \& Jia, 2011), and goal conflict (Kotabe \& Hofmann, 2015) (see also Hirt, Clarkson, \& Jia, 2016).

2. Neither trait anxiety nor gender impacted the results across experiments and are therefore not discussed further.

3. For those interested, removing trait anxiety and gender from the model does not impact the documented effects in either experiment.

4. The pattern of findings across experiments does not change as a function of analyzing the difference score or time 2 anxiety scores controlling for time 1 anxiety scores.

5. Interestingly, we observed limited evidence that resource availability may affect the initial experience of anxiety. In Experiment 1, the high resource availability condition not exposed to any stimulus (i.e., the control) experienced a marginally greater anxiety increase from the baseline to the induction measure, $t(135)=1.81, p=.072$. This finding hints at agreement with a previous neuroimaging finding in which the lack of available mental resources increased activation in the amygdala in response to negative stimuli (Wagner \& Heatherton. 2013). 
Figure 1. Anxiety reduction as a function of resource availability and reward processing (music exposure) in Experiment 1. Note: The Y-axis reports the change in mean STAI-S scores for (Time 1-Time 2) anxiety, and standard error bars are included.

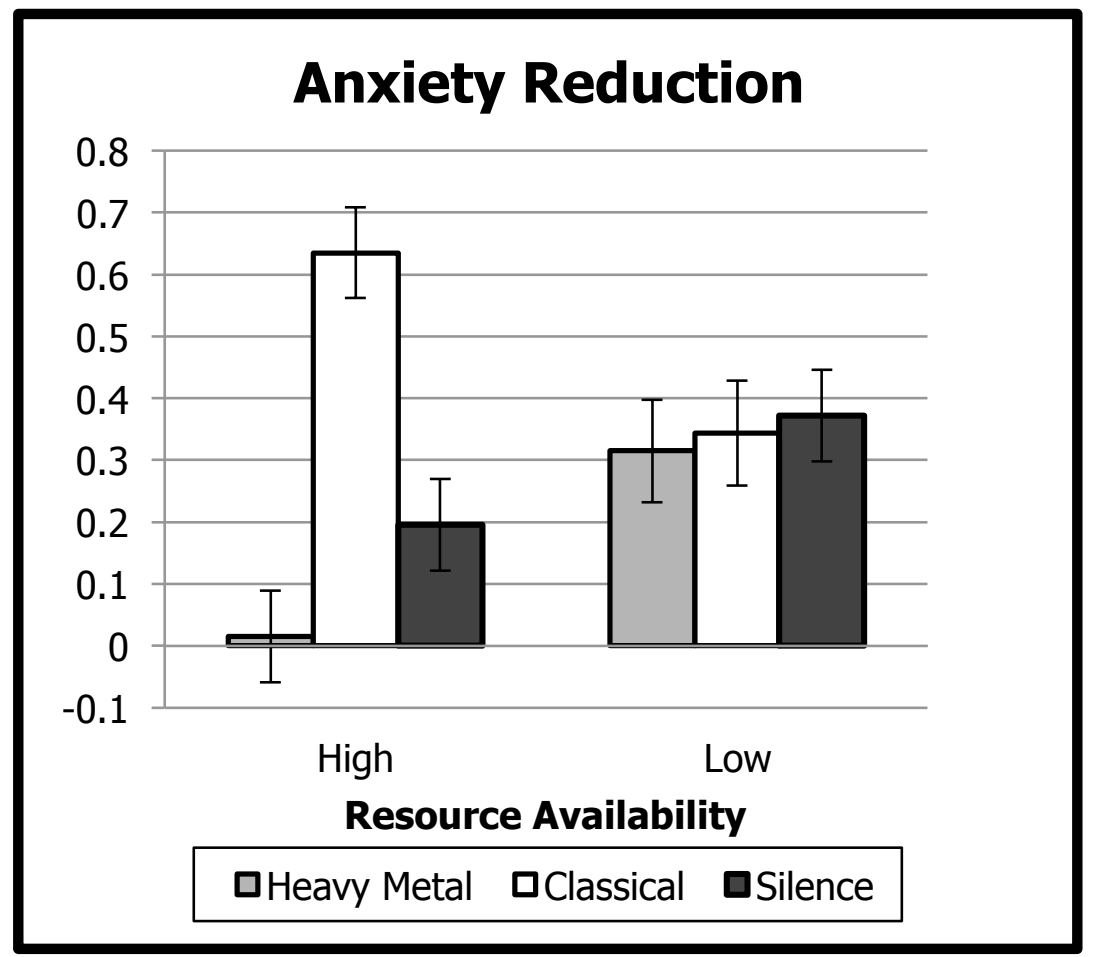


Figure 2. Anxiety reduction as a function of resource availability and reward processing (music exposure) when exposed to high reward intensity (left panel) or low reward intensity (right panel) in Experiment 2. Note: The Y-axis reports the change in mean STAI-S scores for (Time 1 Time 2) anxiety, and standard error bars are included.

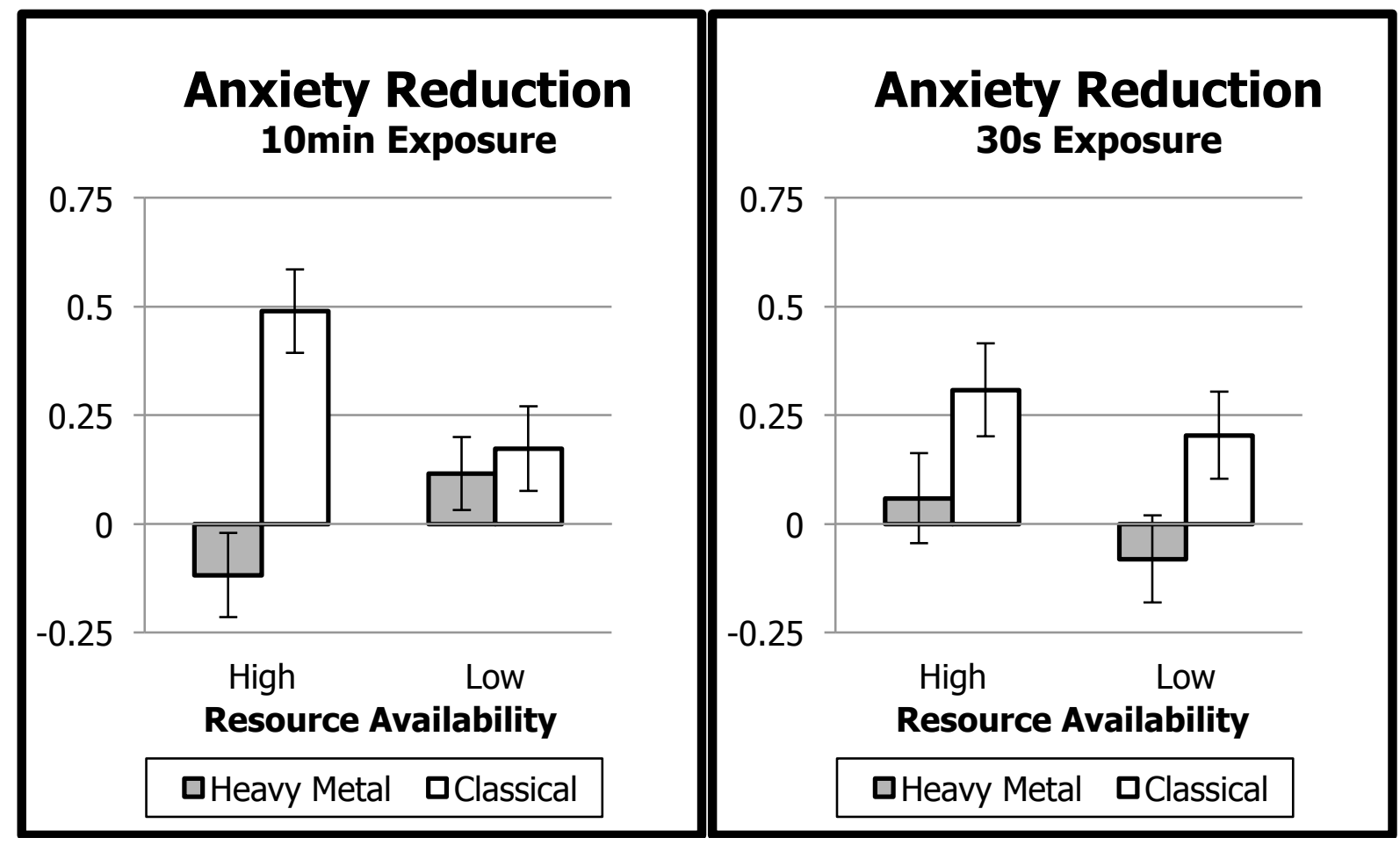


Appendix A. Pilot study exploring the effects of perceived resource availability and reward processing on anxiety reduction.

\section{Pilot Study}

We conducted a pilot study that assessed the interactive effect of perceived resource availability and reward processing on anxiety reduction, given prior research demonstrating that resource availability is altered by individuals' perceptions of mental fatigue (Clarkson, Hirt, Chapman, \& Jia, 2011; Egan, Clarkson, \& Hirt, 2015).

\section{Method}

Sixty-seven undergraduates $\left(M_{\mathrm{age}}=19.78,73 \%\right.$ female $)$ participated for course credit and were randomly assigned to reward conditions. Upon entering the lab and completing background materials, participants indicated their current level of mental fatigue on the four-item mental fatigue subscale of the Multidimensional Fatigue Inventory (Smets, Garssen, Bonke, \& De Haes, 1995). This measure served as our index of resource availability, as subjective perceptions of mental fatigue have been shown to serve as a natural proxy for resource availability (Clarkson et al., 2011). Consistent with prior recommendations for highly skewed data (MacCallum, Zhang, Preacher, \& Rucker, 2002), a median split (Med =2) was used to classify participants as either high or low in cognitive control.

We then induced situational anxiety in all participants by presenting them with information about free will and determinism and informing them that they would be giving a five-minute speech stating their own opinion on the topic. To heighten anxiety, participants were further told that (1) they had three minutes to read over the information and prepare their thoughts before their speech, and (2) they would be videotaped so their speech could later be evaluated for competency. After three minutes, participants recorded their five-minute speech 
using the computers' iMovie function (Connelly \& Denney, 2007). We then assessed participants' state anxiety using the state anxiety subscale of the STAI-Y (Spielberger et al., 1970). The subscale focuses on the extent to which individuals are currently experiencing anxiety-related symptoms. Responses were obtained on 4-point scales labeled 1 -almost never, 2 -sometimes, 3 -often, and 4 -almost always and averaged such that higher scores indicated greater initial anxiety.

Following the anxiety-induction, participants were randomly assigned to listen to one of two music samples as our manipulation of reward. Specifically, participants listened to tenminute clips of either classical or heavy metal music as described in Experiment 2. Afterwards, participants again completed the state anxiety subscale of the STAI-Y before being debriefed and thanked for their participation.

\section{Results}

An anxiety reduction score was created by subtracting participants' post-music state anxiety from their pre-music state anxiety. Higher values indicated greater reduction in anxiety. The anxiety reduction score was submitted to a 2 (Perceived Resource Availability: High or Low) $\times 2$ (Music: Classical or Heavy Metal) ANOVA, with trait anxiety and gender $(73 \%$ female) as covariates. The analysis revealed a significant main effect of music $(F(1,61)=7.69, p$ $<.01$ ); consistent with previous research (Labbé, et al., 2007), greater anxiety reduction was observed following the classical (versus heavy metal) music. Additionally, the analysis revealed a significant main effect of perceived cognitive control $(F(1,61)=9.96, p<.01)$; greater anxiety reduction was observed for those high (versus low) in perceived resource availability. However, these main effects were qualified by a perceived resource availability $\times$ music interaction $\left(F(1,61)=4.40, p<.05, \eta_{p}^{2}=.16\right)$. As illustrated in Figure S1, participants high in perceived 
resource availability showed greater anxiety reduction after exposure to the classical (versus heavy metal) music $(\mathrm{F}(1,37)=11.18, p<.001)$. For those low in perceived resource availability, music exposure had no effect on anxiety reduction $(F<1)$.

\section{Discussion}

These results, though correlational, offered initial evidence of an interaction between resource availability and reward processing in facilitating emotional regulation.

\section{References}

Clarkson, J.J., Hirt, E.R., Chapman, D.A., \& Jia, L. (2011). The impact of illusory fatigue on executive control: Do perceptions of depletion impair working memory capacity? Social Psychological and Personality Science, 2, 231-238.

Connelly, M., \& Denney, D. R. (2007). Regulation of emotions during experimental stress in alexithymia, Journal of Psychosomatic Research, 62, 649-656.

Egan, P.M., Clarkson, J.J., \& Hirt, E.R. (2015). Revisiting the restorative effects of positive mood: An expectancy-based approach to self-control restoration. Journal of Experimental Social Psychology, 57, 87-99.

MacCallum, R.C., Zhang, S., Preacher, K.J., \& Rucker, D.D. (2002). On the practice of dichotomization of quantitative variables. Psychological Methods, 7, 19-40.

Smets, E.M.A., Garssen, B., Bonke, B., De Haes, J.C.J. (1995). The multidimensional fatigue inventory (MFI) psychometric qualities of an instrument to assess fatigue. Journal of Psychosomatic Research, 39, 315-325.

Spielberger, C. D., Gorsuch, R.L., \& Lushene. R.E. (1970). Manual for the State-Trait Anxiety Inventory. Palo Alto, CA: Consulting Psychologists. 
Supplementary Figure 1. Anxiety reduction as a function of perceived resource availability and reward processing (music exposure) in Pilot Study. Note: The Y-axis reports the change in mean STAI-S scores for (Time 1-Time 2) anxiety, and standard error bars are included.



